\title{
Commentary on a special issue: Davydov's approach in the XXI century
}

\author{
Alf Coles ${ }^{1}$ \\ Accepted: 17 December 2020 / Published online: 25 January 2021 \\ (C) The Author(s) 2021
}

\begin{abstract}
The articles in this special issue, collectively, provide overwhelming evidence that a curriculum which has as its primary basis the counting of discrete objects (and hence which introduces numbers as discrete) is not an effective or rational organisation. In this commentary, I discuss the contribution of each article to an understanding of Davydov's ontology and epistemology, issues around the transposition of a pedagogy or curriculum from one country to another, early algebra and proportional or multiplicative reasoning. From Davydov's own research and the writing in these articles, we know children are able to understand abstract structures from an early age. The thinking in this special issue provides tools to investigate and question any context in which such understanding is not routinely taking hold.
\end{abstract}

Keywords Davydov $\cdot$ Early algebra $\cdot$ Proportional reasoning

\section{Introduction}

This is a timely and significant special issue. A key question in the articles, and a prime concern of Davydov's, is: what method of introducing concepts will lead the child to develop the concepts of the research mathematician and, moreover, to develop as a theoretical thinker? I believe these issues remain open research questions of the highest importance. At the same time as suggesting they are open, some clear pointers can be surmised from the articles in this special issue. One conclusion is that there is overwhelming evidence a curriculum which has as its primary basis the counting of discrete objects (and hence which introduces numbers as discrete) is not an effective or rational organisation. This is a conclusion with implications for many countries, and I suspect may sound heretical to some, given there is so much established orthodoxy about the significance of using number to answer discrete, "how many" type questions. However, I believe it is a conclusion that needs to be looked at seriously by policy

Alf Coles

alf.coles@bris.ac.uk 
makers, curriculum designers and text-book writers, as well as one meriting further research. A further conclusion is that it is possible to arrange learning activity so that children move from considering general structures first, to later considering particular instances, and that there are significant benefits to learners in this organisation. A further theme that rippled through my reading of each article was the question of whether there are any universals about human learning of mathematics.

We do know there are several divides or didactical cuts in the school mathematics curriculum which research, from across the world, suggests can be problematic for large numbers of children. Two such divides are the move into algebra and the move into proportional reasoning. Davydov's curriculum is held up by its advocates as a solution to both problems, through having algebra and proportional reasoning around from the start of schooling. I will consider the special issue article contributions to thinking about both these divides. However, before getting to these direct applications of the theory, I begin with two more general considerations, the first relating to the epistemological and ontological commitments in play within Davydov's work and the second relating to issues of transposition of the theory to different contexts.

\section{Epistemology and ontology}

A current sense I have of epistemology and ontology is to read them as accounts of change and stasis, i.e., how it is possible that some things endure (such as the essences of objects) and at the same time that some things change (such as the appearances of objects). And one key question for philosophers is whether there is a sharp distinction, or not, between essences and appearances (in terms what they are - an ontological issue - and how we come to know them, an epistemological issue). I will draw on Radford's (2020) article in considering Davydov's views.

Davydov commits to a relational ontology, in which an object's essence is its internal relations and connections, which allow it also to come into relation to other objects and which are made manifest through activity. Such relations, an object's mediated, reflected, essential being, are the substance of theoretical thought. Theoretical thought, therefore, is an idealisation of practical activity involving objects and of the reproduction in that activity of the universal forms of things, their measures and their laws. A relational ontology is in line with thinkers such as Deleuze, Whitehead and Stengers, who are part of the turn to ontology in the social sciences. On the opposite philosophical fence sit contemporary thinkers such as Bogost (2012), and Harman (2012) who proposes an object-oriented ontology, in which the essences of objects are always withdrawn from human (and non-human) knowing. Harman proposes several critiques of relational ontology, one of which is a challenge of how it can account for identity. If an object's essence is its relations, and every object is ultimately connected to every other object, then how do we ever draw boundaries or establish the identity of an essence?

For Davydov, generalisation is a process of coming to know, through practical activity, an object's essence (i.e., its relations) and coming to know them theoretically. Davydov was influenced by Vygotsky's notion of generalisation and a dialectic logic emphasising movement, evolution and change. Phenomena (including the phenomenon of generalisation) need to be studied as processes, with origins and a history. Subject and object are entangled and coproduced, rather than pre-existing. Many contemporary perspectives would agree at this point, about moving away from a focus on identity (of subject, of object) towards a focus on 
difference or relation (between subject and object). In other words, to take seriously the entanglement of subject and object, I cannot think about them as separate identities brought into contact through an encounter. Rather it is in the encounter that subject and object are brought into being. And yet this conclusion can seem so counter to an experience of making a conscious act or decision, in which our own subjectivity appears primary, pre-existing and continuous. Perhaps the question "who acts?", which can lead us to the identification of the subject, needs to be replaced by the question of "how is it that such a subject is able to act in this way?" (Benjamin, 2015, p. 87). The latter question points to the always-already existing web of relations that allow the possibility of acting. And, significantly, for Davydov, the web of relations has been constituted culturally and historically, rather than merely contingently. Subjectivity is an after-effect of the cultural and historical relations that allow its emergence.

And at this point, I find myself returning to question the very distinction between an object's essence and its appearance. Yusoff (2019) has linked the geological distinction between type and property to a logic that leads to the categorisation of humans into races, classes, and, ultimately, the colonial separation of (white) humans from all others, who are cast as inhuman. A relational ontology ends up disrupting the distinction between essence (type, stasis) and appearance (property, change). Benjamin (2015) suggests that the only way to make sense of a relationality as being primary is that what pre-exists objects are plural relations, from which individuals become differentiated. For Davydov, the essential relations of an object are reproducible mentally, through reflection on practical activity - this being what it means to know something theoretically. Radford (2020) argues for a more "poetic" view, still within the spirit of Davydov's thinking, but one in which "a concept would be what we enact with others in joint activity", taking us beyond the theoretical and scientific and inclusive of aesthetics, ethics, politics and affect. I have found it helpful to bring the sense of Radford's expansion of what concepts are, and how we come to know them, to the reading of the other articles in the special issue.

\section{Transposing a curriculum}

Davydov developed his ideas in the time of the Soviet Union. One of the deep questions addressed, across the special issue, relates to the transposition of theory and curriculum from one context to another and the extent to which background ontological and epistemological commitments match across those contexts (Mellone, Ramploud, \& Carotenuto, 2020). Mellone et al. propose the significance of a process of deconstruction, when encountering teaching practices from a new culture. They implement this process, working with teachers in the Italian system, while engaging in Davydov's curriculum ideas. The process of deconstruction focuses on values and beliefs about mathematics education inherent in any practice and, for Mellone et al., is a mechanism for bringing attention to our own previously unmarked values and beliefs about mathematics education, a process they illustrate powerfully. Several other articles conduct their own version of a process of deconstruction or show evidence of such work having taken place, and I mention three below.

Polotskaia and Savard (2020) have been involved, over several years, in implementing Davydov's ideas in a Canadian elementary school context and have, elsewhere, developed the important distinction between two "paradigms" in which children can learn about number, an "operational paradigm", which is perhaps a traditional one in many contexts, based on teaching arithmetical operations as the basis for learning number and in which number is 
discrete, e.g. in tasks such as one-to-one correspondence and counting. The alternative is a "relational paradigm", which they take from Davydov's work, in which number is introduced first as a relationship (e.g., in the context of measuring lengths) prior to developing the use of operations with number. While Polotskaia and Savard do not consider some of the wider values and beliefs called for by Mellone et al., there is a detailed epistemological analysis of multiplicative relationship, which is the focus of their paper, and the whole notion of the relational paradigm, in a sense, captures these researchers' work to make sense of Davydov's ideas in their own context.

One of the most well-known applications of Davydov's curriculum outside Russia is the Measure Up programme, which forms the context of the contribution to this special issue by Venenciano, Yagi, and Zenigami (2020). Their study includes a critique of the ways in which manipulatives can support the learning of mathematics and how physical actions with objects can be linked to mathematical symbols. These authors use the phrase "relational thinking" to describe what they see at the heart of Davydov's approach. The first instance of relational thinking that is leveraged in the Measure Up programme (and Davydov's curriculum) is to consider relations between quantities of greater than, less than and equal to. I have long been fascinated in the similarity between this starting point and Gattegno's curriculum (1974), which starts with play with Cuisenaire rods, first codified in terms of precisely those relations, with a focus on length. Venenciano et al. (2020) analyse the activities of students within the Measure Up programme, partly drawing on work I have done with Nathalie Sinclair, in relation to the notion of a symbolically structured environment (Coles \& Sinclair, 2019). One of the features of symbolically structured environments is that symbols are introduced to represent actions or relationships. Teaching may begin with concrete materials, as advocated by many, and if the focus, from the start, can be on relationships between the materials, then students do not have to grapple with competing systems of thought (one for the materials and one for the symbols) and translations between them. Rather, the symbols can be introduced to stand for actions on objects, which can become evoked virtually, or mentally, by those symbols. And, crucially, actions can be done and undone-so by focusing symbolisation on actions, we immediately make inverse operations and relations simultaneously available. If one length is greater than another, children can reason that the other is smaller than the first and, in time, that if $\mathrm{A}+\mathrm{C}=\mathrm{B}$, then $\mathrm{B}-\mathrm{C}=\mathrm{A}$.

Another article of the Special Issue which considers a transposition of Davydov's theory to a new context is Venkat, Askew, and Morrison's (2020) study, involving the application of ideas about early number to a South African context. These authors are explicit about the "shape-shifting" needed to find ways of working applicable to a schooling system that is in the after-life of apartheid, still haunted by the disastrous effects of segregation on the education system. The key distinction they draw is between calculating-by-counting (which they characterise as the established practice in South Africa) and calculating-by-structuring (which is their transposition of Davydov's insight).

The authors cited above develop their own ways of making sense of Davydov, relevant to their own contexts, invoking a relational paradigm, relational thinking and calculating-by-structuring. And across these transpositions, a commonality is the attention to mathematical concepts themselves as relations and the pedagogic advantage of offering mathematics to children by way of bringing attention to relationship first, before coming to consider particulars. There can be attention to relationships between lengths, and relationships between numbers, before considering lengths and numbers in 
themselves. The implementation of this idea is strikingly different to the curricula I have worked within, e.g., in England, and appears to have the potential to be transformative. The successful outcomes reported across countries as diverse as Russia, Italy, Canada and South Africa point to some potentially general findings, alongside the importance of culturally sensitive adaptations.

\section{Algebra in the elementary curriculum}

Venkat et al. (2020) distinguish between transpositions of Davydov's work which attend to structure and use algebraic representations to symbolise relations and approaches which attend to structure but where numbers are used to symbolise relations. It is evidently possible to engage deeply in Davydov's work and transpose it to a different context, while leaving behind the algebra present in the original curriculum. It is perhaps an extension of the insight that we can helpfully present children with mathematical relationships before getting them to work on mathematical particulars, or objects, that leads to the potential to use algebra to express those relationships. If the move into algebra proves to be an obstacle for children worldwide, then a curriculum in which algebra is introduced before arithmetic would potentially smooth the path of future learning.

Two articles in this special issue explicitly address the issue of early algebra, with which Davydov's work is often associated. Freiman and Fellus (2020) provide highly relevant historical context to Davydov's thinking (a form of deconstruction work called for by Mellone et al., 2020) in order to relate the curriculum proposals to wider issues in the teaching and learning of algebra. Their article provides a detailed analysis of what is meant by the ascent from the abstract to the concrete - a phrase that is perhaps one of the best known of Davydov's - and perhaps also one that is easily misunderstood. Freiman and Fellus point to the manner in which the curriculum begins with children engaging in a consideration of general relationships (the abstract), thereby helping children develop a capacity for theoretical thinking, before they engage in solving particular (concrete) problems. Again, in common with Gattegno's (1974) curriculum, algebra precedes arithmetic. And it is the contention of Davydov and his followers that proceeding in this manner, from theoretical to practical, is a more effective way of learning mathematics than the alternative of moving from particular to general. (Gattegno used the word "economical" for the advantages of his curriculum, referring to the use of children's time.)

Eriksson and Eriksson (2020) conducted a study demonstrating one example of "effectiveness", which was the manner in which a Davydov-inspired learning activity led to generalisation and an appreciation of general structures, by young children in a multi-lingual context. The manner in which symbols arise from activity within Davydov's work potentially offers huge advantages in multi-lingual classrooms, and I would see this as a rich area of future research. The sense of beginning with tasks which generate a need for new theoretical knowledge mirrors a challenge from Sutherland (1991):

Can we develop a school algebra culture in which pupils find a need for algebraic symbolism to express and explore their mathematical ideas? (p. 46)

It is evident that Davydov's curriculum provides one positive response to Sutherland's question. 


\section{Proportion in the elementary curriculum}

A second well-recognised discontinuity in children's learning of mathematics concerns the move into proportional reasoning. In the same way that Davydov's curriculum introduces early algebra as a way of overcoming later difficulties of the move from arithmetic to algebra, the curriculum also introduces number as a proportion, from the very start, hence potentially nullifying the obstacle of the need for a later move from additive to multiplicative, or proportional, reasoning. Davydov's curriculum, in relation to algebra and proportional reasoning, offers a conceptual continuity since children are introduced, from the very start, to a general formulation that gets specified in greater and greater detail but never needs to be rejected. Two articles from the special issue (Polotskaia \& Savard, 2020; Vysotskaya, Lobanova, Rekhtman, \& Yanishevskaya, 2020) consider questions of proportional or multiplicative reasoning (which I take to be synonymous).

There have been several important categorisations of multiplicative thinking, not just within Davydov's writing, and Polotskaia and Savard (2020) survey this work in arriving at the following three types, in each case emphasising there are three elements: (1) multiplicative composition relationship, "where one quantity is composed of a number of equal parts", for example, a box contains 5 oranges, if I have 3 boxes, there are 15 oranges. The unit here is a box of oranges, or the measure 5 oranges per box. The coefficient is the number of boxes, giving a result of a number of oranges; (2) multiplicative comparison relationship where "one quantity is compared in a multiplicative way. The latter is physically distinct from the former", for example, the length of a red rod fits 5 times into the length of an orange rod. The red rod acts as a unit, with " 5 " having the role of coefficient; (3) Cartesian product relationship where "all three elements have different physical origins and none of them can be seen as a pure number or as a unit of measurement", for example, a rectangle has sides $3 \mathrm{~cm}$ and $5 \mathrm{~cm}$, so its area is $15 \mathrm{~cm}^{2}$. Each side length plays the role of a dimension, and their product is the area.

Polotskaia and Savard's aim is to articulate the minimal set of multiplicative thinking tools, to cover the Canadian elementary curriculum. This aim reminds me of a challenge the UKbased mathematics educator, Dick Tahta, would sometimes talk about: what are the canonical images of mathematics, a minimal set, that might be sufficient for the entire secondary curriculum as well? Vysotskaya et al. (2020) also consider the role of proportional reasoning, and they are concerned with the critique of Simon and Placa (2012) which is that while Davydov's curriculum introduces several important forms of proportional reasoning, it does not give children access to reasoning about intensive quantities (defined below). Vysotskaya et al. (2020) agree with this critique and find, within Davydov's writings, suggestions for a consideration of intensive quantities which they take up and develop.

Intensive quantities are those which cannot be measured directly, for example, speed would be an intensive quantity, whereas distance and time, both being directly measurable, are extensive quantities. Simon and Placa (2012) make the distinction, however, between intensive quantities that can be treated extensively, by effectively ignoring the rate, through taking it as being "per one". So, in the case of boxes holding 5 oranges, although this may appear to have the form of an intensive quantity (e.g. "5 oranges per box") since the "per box" in effect is operationalised as a "per one unit", what looks like an intensive quantity is one that can actually be measured directly and hence is extensive. Simon and Placa (2012) conclude that a consistent representation of intensive quantities may not be possible. What Vysotskaya et al.'s (2020) research sets out to do is to test the proposition of whether work on intensive quantities can be made accessible to first grade students, as an addition and extension of Davydov's 
curriculum and via a consideration of what might be considered to be ratio problems. I see it as an open and important question, linked to research on student understanding of co-variation, as to whether Polotskaia and Savard's minimal list needs expansion as a result of these considerations.

\section{Conclusion}

Schmittau's work in bringing Davydov's thinking to the attention of an English-speaking audience is fittingly commemorated in a tribute in this special issue. Davydov's curriculum surely challenges all mathematics educators to re-consider the potential for mathematics education to develop theoretical, critical thinking. As the conclusion of the tribute states "work must still be done to learn from lessons embedded in the Davydov curriculum."

I am writing this commentary at the end of 2020 when threats of climate change, pandemic outbreak, environmental depletion, biodiversity loss and far right politics, to name just a few challenges, threaten the very presence of life on the planet. A question I sense many mathematics educators are asking is what role learning mathematics can have in terms of preparing children to face the kind of world we are leaving them. Davydov offers one answer, that the purpose of mathematics education is in the development of the ability to think theoretically. And within such theoretical thinking is the capacity to consider general structures and a confidence in symbolising relationships, surely relevant and potentially vital skills for the future. My strong hope is that this special issue provokes renewed interest in the potential of Davydov's ideas to inform curriculum innovation. We know children are able to understand abstract structures from an early age; the thinking in the special issue provides tools to investigate and question any context in which such understanding is not routinely taking hold.

Open Access This article is licensed under a Creative Commons Attribution 4.0 International License, which permits use, sharing, adaptation, distribution and reproduction in any medium or format, as long as you give appropriate credit to the original author(s) and the source, provide a link to the Creative Commons licence, and indicate if changes were made. The images or other third party material in this article are included in the article's Creative Commons licence, unless indicated otherwise in a credit line to the material. If material is not included in the article's Creative Commons licence and your intended use is not permitted by statutory regulation or exceeds the permitted use, you will need to obtain permission directly from the copyright holder. To view a copy of this licence, visit http://creativecommons.org/licenses/by/4.0/.

\section{References}

Benjamin, A. (2015). Towards a relational ontology: Philosophy's other possibility. Albany, NY: State University of New York Press.

Bogost, I. (2012). Alien phenomenology, or what it's like to be a thing. Minneapolis, MN: University of Minnesota Press.

Coles, A., \& Sinclair, N. (2019). Re-thinking 'concrete to abstract': Towards the use of symbolically structured environments. Canadian Journal of Science, Mathematics, and Technology Education, 19(4), 465-480.

Eriksson, H., \& Eriksson, I. (2020). Learning actions indicating algebraic thinking in multilingual classrooms. Educational Studies in Mathematics. Advanced online publication. https://doi.org/10.1007/s10649-020$10007-y$.

Freiman, V., \& Fellus, O. (2020). Closing the gap on the map: Davydov's contribution to current early algebra discourse in light of the 1960s Soviet debates over word-problem solving. Educational Studies in Mathematics. Advanced online publication. https://doi.org/10.1007/s10649-020-09989-6.

Gattegno, C. (1974). The common sense of teaching mathematics. New York, NY: Educational Solutions. 
Harman, G. (2012). The well-wrought broken hammer: Object-oriented literary criticism. New Literary History, 43(2), 183-203. Retrieved December 1, 2020, from http://www.jstor.org/stable/23259371

Mellone, M., Ramploud, A., \& Carotenuto, G. (2020). An experience of cultural transposition of the El'koninDavydov curriculum. Educational Studies in Mathematics. Advanced online publication. https://doi.org/10. 1007/s10649-020-09942-7

Polotskaia, E., \& Savard, A. (2020). Some multiplicative structures in elementary education: A view from relational paradigm. Educational Studies in Mathematics. Advanced online publication. https://doi.org/10. 1007/s10649-020-09979-8

Radford, L. (2020). Davydov's concept of the concept and its dialectical materialist background. Educational Studies in Mathematics. Advanced online publication. https://doi.org/10.1007/s10649-020-09959-y

Simon, M. A., \& Placa, N. (2012). Reasoning about intensive quantities in whole-number multiplication? A possible basis for ratio understanding. For the Learning of Mathematics, 32(2), 35-41.

Sutherland, R. (1991). Some unanswered research questions on the teaching and learning of algebra. For the Learning of Mathematics, 11(3), 40-46.

Venenciano, L., Yagi, S., \& Zenigami, F. (2020). The development of relational thinking: A study of Measure Up first-grade students' thinking and their symbolic understandings. Educational Studies in Mathematics. Advanced online publication. https://doi.org/10.1007/s10649-020-10014-z

Venkat, H., Askew, M., \& Morrison, S. (2020). Shape-shifting Davydov's ideas for early number learning in South Africa. Educational Studies in Mathematics. Advanced online publication. https://doi.org/10.1007/ s10649-020-09993-w

Vysotskaya, E., Lobanova, A., Rekhtman, I. \& Yanishevskaya, M. (2020). The challenge of proportion: Does it require rethinking of the measurement paradigm? Educational Studies in Mathematics. Advanced online publication. https://doi.org/10.1007/s10649-020-09987-8

Yusoff, K. (2019). A billion black Anthropocenes or none. Minneapolis, MN: University of Minnesota Press.

Publisher's note Springer Nature remains neutral with regard to jurisdictional claims in published maps and institutional affiliations. 